\title{
EIGENVALUE BOUNDS FOR SCHRÖDINGER OPERATORS WITH COMPLEX POTENTIALS. II
}

\author{
RUPERT L. FRANK AND BARRY SIMON
}

\begin{abstract}
Laptev and Safronov conjectured that any non-positive eigenvalue of a Schrödinger operator $-\Delta+V$ in $L^{2}\left(\mathbb{R}^{\nu}\right)$ with complex potential has absolute value at most a constant times $\|V\|_{\gamma+\nu / 2}^{(\gamma+\nu / 2) / \gamma}$ for $0<\gamma \leq \nu / 2$ in dimension $\nu \geq 2$. We prove this conjecture for radial potentials if $0<\gamma<\nu / 2$ and we 'almost disprove' it for general potentials if $1 / 2<\gamma<\nu / 2$. In addition, we prove various bounds that hold, in particular, for positive eigenvalues.
\end{abstract}

\section{INTRODUCTION AND MAIN RESULTS}

In this paper we are interested in eigenvalues of Schrödinger operators

$$
-\Delta+V \quad \text { in } L^{2}\left(\mathbb{R}^{\nu}\right)
$$

with (possibly) complex-valued potentials $V$. More precisely, we want to derive bounds on the location of these eigenvalues assuming only that $V$ belongs to some $L^{p}\left(\mathbb{R}^{\nu}\right)$ with $p<\infty$. This assumption, for suitable $p$, will also guarantee that $-\Delta+V$ can be defined via the theory of $m$-sectorial forms. Also, $p<\infty$ implies that eigenvalues outside of $[0, \infty)$ are discrete and have finite algebraic multiplicities.

If $V$ is real-valued (so that discrete eigenvalues are negative), it is a straightforward consequence of Sobolev inequalities that

$$
|E|^{\gamma} \leq C_{\gamma, \nu} \int_{\mathbb{R}^{\nu}}|V|^{\gamma+\nu / 2} d x
$$

for every $\gamma \geq 1 / 2$ if $\nu=1$ and every $\gamma>0$ if $\nu \geq 2$. Here $C_{\gamma, \nu}$ is a constant independent of $V$. For this bound, see [15, 19] and also [4] for optimal constants, optimal potentials and stability results.

The question becomes much more difficult if $V$ is allowed to be complex-valued. Laptev and Safronov [18] conjectured that for any $\nu \geq 2$ and $0<\gamma \leq \nu / 2$ there is a $C_{\gamma, \nu}$ such that (1.1) holds for all eigenvalues $E \in \mathbb{C} \backslash[0, \infty)$. Prior to their conjecture, Abramov, Aslanyan and Davies [1] (see also [5]) had shown this for $\nu=1$ and $\gamma=1 / 2$. In [8] the Laptev-Safronov conjecture was proved for $\nu \geq 2$ and $0<\gamma \leq 1 / 2$.

In this paper we accomplish the following:

(c) 2015 by the authors. This paper may be reproduced, in its entirety, for non-commercial purposes.

Work partially supported by U.S. National Science Foundation grants PHY-1347399, DMS-1363432 (R.L.F.) and DMS-1265592 (B.S.). 
(A) We almost disprove the Laptev-Safronov conjecture for $\nu \geq 2$ and $1 / 2<\gamma<$ $\nu / 2$ (Theorem 2.1).

(B) We prove the Laptev-Safronov conjecture for radial potentials for $\nu \geq 2$ and $1 / 2<\gamma<\nu / 2$

(C) We give a simple proof that for $0<\gamma \leq 1 / 2$ the bound (1.1) holds also for eigenvalues $E \in[0, \infty)$. (We note that a deep result of Koch-Tataru [17] shows that, in fact, there are no positive eigenvalues.)

(D) We prove an eigenvalue bound for $V \in L^{\gamma_{1}+\nu / 2}\left(\mathbb{R}^{\nu}\right)+L^{\gamma_{2}+\nu / 2}\left(\mathbb{R}^{\nu}\right)$ with $0<$ $\gamma_{1}<\gamma_{2} \leq 1 / 2$ if $\nu=2$ and $0 \leq \gamma_{1}<\gamma_{2} \leq 1 / 2$ if $\nu \geq 3$

By 'almost disprove' in (A) we mean we construct a sequence of real-valued potentials $V_{n}$ such that $-\Delta+V_{n}$ has eigenvalue 1 but $\left\|V_{n}\right\|_{p} \rightarrow 0$ for any $p>(1+\nu) / 2$. If Laptev and Safronov had formulated their conjecture for any eigenvalue $E \in \mathbb{C}$ (and not only for $E \in \mathbb{C} \backslash[0, \infty)$ ), we would have disproved it. In particular, this is interesting in view of $(\mathrm{C})$, where we prove that for $0<\gamma \leq 1 / 2$ the conjecture holds in fact also for eigenvalues in $[0, \infty)$. Note that if we were able to show that the eigenvalue 1 of $-\Delta+V_{n}$ becomes a non-real eigenvalue of $-\Delta+V_{n}+\varepsilon W$ for some nice $W$ (say with $\operatorname{Im} W \geq 0)$ and $\varepsilon$ small, we could also disprove the conjecture.

Our construction of the potentials $V_{n}$ in the proof of Theorem 2.1] is inspired by a construction of Ionescu and Jerison [14]. Using ideas of Wigner and von Neumann [35] (see also [27, Section XIII.13]) we are able to simplify their construction.

We also prove (Theorem 2.2) that a bound of the form (1.1) cannot hold, even for radial potentials, if $\gamma>\nu / 2$. Of course, Laptev and Safronov conjectured such a bound only for $\gamma<\nu / 2$, but the fact that this is the correct upper bound is not obvious. Our construction extends the Wigner-von Neumann construction [35] (see also [27]) to arbitrary dimension $\nu$, which is interesting in its own right. Our counterexamples are constructed in Section 2. In passing we mention that while the Wigner-von Neumann example has been studied extensively, we are not aware of similar results about the Ionescu-Jerison example. It would be interesting to extend the results of Naboko [22] and Simon [29] on dense embedded point spectrum based on the Wigner-von Neumann example to instead use the Ionescu-Jerison example.

Concerning (B), we recall that the proof in [8] of (1.1) for $0<\gamma \leq 1 / 2$ relied on uniform Sobolev bounds due to Kenig-Ruiz-Sogge [16], namely,

$$
\left\|(-\Delta-z)^{-1} f\right\|_{p^{\prime}} \leq C|z|^{-\nu / 2+\nu / p-1}\|f\|_{p}, \quad 2 \nu /(\nu+2)<p \leq 2(\nu+1) /(\nu+3),
$$

with $C$ independent of $z$ and with $p^{\prime}=p /(p-1)$. (In [16] this bound is only proved for $\nu \geq 3$, but the same argument works for $\nu=2$ as well, see [8].) The range of exponents $2 \nu /(\nu+2)<p \leq 2(\nu+1) /(\nu+3)$ in (1.2) corresponds to $0<\gamma \leq 1 / 2$ in (1.1). Bounds of the form (1.2) cannot hold for exponents $2(\nu+1) /(\nu+3)<p<2 \nu /(\nu+1)$ (corresponding to $1 / 2<\gamma<\nu / 2$ ). However, as we shall show (Theorem 4.3), they do hold if one replaces the space $L^{p}\left(\mathbb{R}^{\nu}\right)$ by $L^{p}\left(\mathbb{R}_{+}, r^{\nu-1} d r ; L^{2}\left(\mathbb{S}^{\nu-1}\right)\right)$ and similarly for $L^{p^{\prime}}\left(\mathbb{R}^{\nu}\right)$. In fact, these bounds prove (1.1) not only for radial potentials, but for 
general potentials in $L^{\gamma+\nu / 2}\left(\mathbb{R}_{+}, r^{\nu-1} d r ; L^{\infty}\left(\mathbb{S}^{\nu-1}\right)\right)$ with the obvious replacement on the right side; see Theorem 4.1. We also prove a Lorentz space result at the endpoint $\gamma=\nu / 2 ;$ see Theorem 4.2 ,

Our results for $1 / 2<\gamma \leq \nu / 2$ are based on arguments by Barcelo, Ruiz and Vega [2] and, in particular, precise bounds on Bessel functions. This is further discussed in Section 4 and in the appendix.

We prove (C) in Section [3. Our argument is based on (1.2), like that in [8], but is more direct and avoids Birman-Schwinger operators. As we mentioned above, the deep results of Koch and Tataru [17] imply that $-\Delta+V$ has no positive eigenvalues if $V \in L^{\gamma+\nu / 2}\left(\mathbb{R}^{\nu}\right)$ with $0<\gamma<1 / 2$; see also [14 for the case $\gamma=0$ in dimensions $\nu \geq 3$. (The fact that the results of [17] apply also to complex-valued potentials is not emphasized there, but is clear from their proof strategy via Carleman inequalities. Also, the fact that $V \in L^{\gamma+\nu / 2}\left(\mathbb{R}^{\nu}\right)$ satisfies Assumption A.2 in [17 for $\gamma$ as above can be easily verified using Sobolev embedding theorems; see, for instance, the proof of Lemma 3.5 in [10].)

We include our proof of $(\mathrm{C})$ since it is much simpler than the arguments in [14, 17] and since the same reasoning will give the assertion in (B) for $E \in[0, \infty)$ where the results of [17] are not applicable.

The bounds mentioned in (D), see Theorem 3.4 , are new, even for $E \in \mathbb{C} \backslash[0, \infty)$. They are also derived from (1.2). Somewhat related bound in $\nu=1$ are contained in [5].

In this paper we have only discussed bounds on single eigenvalues. The situation for sums of eigenvalues is less understood and we refer to [9, 18, 3, 6, 11] and references therein for results and open questions in this direction. Also, we emphasize that we work only under an $L^{p}$ condition on $V$. In contrast, results under exponential decay assumptions are classical (see, e.g., [23, 20, 21] and also [30, 31]) and extensions to subexponential decay were studied in a remarkable series of papers of Pavlov [24, 25, 26]. For results in the discrete, one-dimensional case we refer, for instance, to [7, 12].

Acknowledgemnts. The authors would like to thank L. Golinskii, H. Koch, A. Laptev, O. Safronov and D. Tataru for helpful corresondence.

\section{Counterexamples}

The following theorem shows, in particular, that the bound (1.1) cannot be valid for positive eigenvalues of Schrödinger operators with real potentials if $\nu \geq 2$ and $\gamma>(\nu+1) / 2$. Our proof simplifies the construction of potentials that appeared in [14] in a different, but related context. 
Theorem 2.1. For any $\nu \geq 2$ there is a sequence of potentials $V_{n}: \mathbb{R}^{\nu} \rightarrow \mathbb{R}, n \in \mathbb{N}$, such that 1 is an eigenvalue of $-\Delta+V$ in $L^{2}\left(\mathbb{R}^{\nu}\right)$ and

$$
\left|V_{n}(x)\right| \leq \frac{C}{n+\left|x_{1}\right|+\left|x^{\prime}\right|^{2}}, \quad x=\left(x_{1}, x^{\prime}\right) \in \mathbb{R} \times \mathbb{R}^{\nu-1},
$$

with $C>0$ independent of $n$. In particular, for any $p>(\nu+1) / 2$,

$$
\left\|V_{n}\right\|_{L^{p}} \rightarrow 0 \quad \text { as } n \rightarrow \infty \text {. }
$$

Proof. We look for an eigenfunction of the form $\psi(x)=w(x) \sin x_{1}$. Then

$$
-\Delta \psi=\psi-2\left(\partial_{x} w\right) \cos x_{1}-(\Delta w) \sin x_{1},
$$

so the eigenvalue equation will be satisfied if we set

$$
V:=2 \frac{\partial_{1} w}{w} \cot x_{1}+\frac{\Delta w}{w} .
$$

We need to choose $w$ in such a way that $\psi \in L^{2}$ and that $V$ satisfies the required bounds. In particular, $\partial_{1} w$ needs to vanish where $\sin x_{1}$ does. In order to achieve this, we set

$$
g\left(x_{1}\right):=4 \int_{0}^{x_{1}} \sin ^{2} y d y=2 x_{1}-\sin \left(2 x_{1}\right)
$$

and

$$
w_{n}(x):=\left(n^{2}+g\left(x_{1}\right)^{2}+\left|x^{\prime}\right|^{4}\right)^{-\alpha} .
$$

The potential $V_{n}$ is defined with $w_{n}$ in place of $w$. The parameter $n$ here is not necessarily an integer, but we do require later that $n \geq 1$. Finally, the parameter $\alpha$ will be chosen so that $w \in L^{2}\left(\mathbb{R}^{\nu}\right)$ (which implies $\psi \in L^{2}\left(\mathbb{R}^{\nu}\right)$ ). Note that

$$
\int_{\mathbb{R}^{\nu}}\left|w_{n}(x)\right|^{2} d x=2\left|\mathbb{S}^{\nu-2}\right| \int_{0}^{\infty}\left(n^{2}+g\left(x_{1}\right)^{2}\right)^{-2 \alpha+(\nu-1) / 2} d x_{1} \int_{0}^{\infty} \frac{r^{\nu-2} d r}{\left(1+r^{4}\right)^{2 \alpha}}
$$

is finite provided $\alpha>\nu / 4$, which we assume in the following. We do not keep track of the dependence of our estimates on $\alpha$.

A quick computation shows that

$$
V_{n}=-\frac{4 \alpha}{m_{n}} g g^{\prime} \cot x_{1}+\frac{4 \alpha(\alpha+1)}{m_{n}^{2}}\left(g^{2}\left(g^{\prime}\right)^{2}+4\left|x^{\prime}\right|^{6}\right)-\frac{2 \alpha}{m_{n}}\left(\left(g^{\prime}\right)^{2}+g g^{\prime \prime}+2(\nu+1)\left|x^{\prime}\right|^{2}\right)
$$

with $m_{n}(x):=n^{2}+g\left(x_{1}\right)^{2}+\left|x^{\prime}\right|^{4}$. Note that $g^{\prime} \cot x_{1}=4 \sin x_{1} \cos x_{1}$ is bounded. Moreover, $|g|,\left|x^{\prime}\right|^{2} \leq m_{n}^{1 / 2}$ and $\left|g^{\prime}\right|,\left|g^{\prime \prime}\right| \leq C$, so

$$
\left|V_{n}\right| \leq C\left(m_{n}^{-1 / 2}+m_{n}^{-1}\right) \text {. }
$$

Using $n \geq 1$, we find $m_{n}^{-1} \leq n^{-1} m_{n}^{-1 / 2} \leq m_{n}^{-1 / 2}$, so $\left|V_{n}\right| \leq C m_{n}^{-1 / 2}$. This bound is equivalent to the one stated in the theorem.

Finally, we note that by scaling

$$
\int_{\mathbb{R}^{\nu}}\left|V_{n}\right|^{p} d x \leq C \int_{\mathbb{R}^{\nu}} \frac{d x}{\left(n+\left|x_{1}\right|+\left|x^{\prime}\right|^{2}\right)^{p}}=n^{-p+(\nu+1) / 2} C \int_{\mathbb{R}^{\nu}} \frac{d x}{\left(1+\left|x_{1}\right|+\left|x^{\prime}\right|^{2}\right)^{p}}
$$


For $p>(\nu+1) / 2$, the right side tends to zero since $\left(1+\left|x_{1}\right|+\left|x^{\prime}\right|^{2}\right)^{-1} \in L^{p}$ in this case. This finishes the proof of the theorem.

We emphasize that the eigenfunctions corresponding to the eigenvalue 1 of $-\Delta+V_{n}$ can have arbitrarily fast or slow (consistent with being square-integrable) algebraic decay in $\left|x_{1}\right|+\left|x^{\prime}\right|^{2}$. We also note that (for fixed $n$ ) the potential $V_{n}$ has the asymptotic behavior

$$
\begin{aligned}
V_{n}(x)= & -\frac{16 \alpha x_{1} \sin ^{2}\left(2 x_{1}\right)}{4\left|x_{1}\right|^{2}+\left|x^{\prime}\right|^{4}}+\frac{16 \alpha(\alpha+1)\left|x^{\prime}\right|^{6}}{\left(4\left|x_{1}\right|^{2}+\left|x^{\prime}\right|^{4}\right)^{2}}-\frac{4 \alpha\left(4 x_{1} \cos \left(2 x_{1}\right)+(\nu+1)\left|x^{\prime}\right|^{2}\right)}{4\left|x_{1}\right|^{2}+\left|x^{\prime}\right|^{4}} \\
& +O\left(\left(\left|x_{1}\right|+\left|x^{\prime}\right|^{2}\right)^{-2}\right)
\end{aligned}
$$

as $\left|x_{1}\right|+\left|x^{\prime}\right|^{2} \rightarrow \infty$.

Our next theorem shows, in particular, that the bound (1.1) cannot be valid for positive eigenvalues of Schrödinger operators with real, radial potentials if $\nu \geq 1$ and $\gamma>1 / 2$. Our proof extends the Wigner-von Neumann construction [35] (see also [27]) to arbitrary dimensions $\nu \geq 1$.

Theorem 2.2. For any $\nu \geq 1$ there is a sequence of radial potentials $V_{n}: \mathbb{R}^{\nu} \rightarrow \mathbb{R}$, $n \in \mathbb{N}$, such that 1 is an eigenvalue of $-\Delta+V$ in $L^{2}\left(\mathbb{R}^{\nu}\right)$ and

$$
\left|V_{n}(x)\right| \leq \frac{C}{n+|x|}, \quad x \in \mathbb{R}^{\nu}
$$

with $C>0$ independent of $n$. In particular, for any $p>\nu$,

$$
\left\|V_{n}\right\|_{L^{p}} \rightarrow 0 \quad \text { as } n \rightarrow \infty \text {. }
$$

Proof. We first observe that we may assume $\nu \geq 2$. Indeed, for $\nu=1$ we simply extend $V_{n}$ from $\nu=3$ to an even function on $\mathbb{R}$. The proof below will show that the corresponding eigenfunction $\psi_{n}$ is radial and we can extend $r \psi_{n}$ to an odd function on $\mathbb{R}$ which will satisfy the correct equation.

Now let $\nu \geq 2$. We look for an eigenfunction of the form

$$
\psi(x)=\varphi(r) w(r), \quad r=|x|,
$$

where $\varphi$ is a radial function solving $-\Delta \varphi=\varphi$ in $\mathbb{R}^{\nu}$ (in particular, $\varphi$ is regular at the origin). It is known that, up to a multiplicative constant, $\varphi(r)=r^{-(\nu-2) / 2} J_{(\nu-2) / 2}(r)$, where $J_{(\nu-2) / 2}$ is a Bessel function. This follows from Bessel's equation

$$
-J_{(\nu-2) / 2}^{\prime \prime}-r^{-1} J_{(\nu-2) / 2}^{\prime}+\left(\frac{\nu-2}{2}\right)^{2} r^{-2} J_{(\nu-2) / 2}=J_{(\nu-2) / 2},
$$

as well as

$$
J_{(\nu-2) / 2}(r) \sim \Gamma(\nu / 2)^{-1}(r / 2)^{(\nu-2) / 2} \quad \text { as } r \rightarrow 0 .
$$

In the following we make use of the asymptotics

$$
J_{(\nu-2) / 2}(r)=\sqrt{\frac{2}{\pi r}} \sin (r-\pi(\nu-3) / 4)+O\left(r^{-3 / 2}\right) \quad \text { as } r \rightarrow \infty,
$$


which may also be differentiated with respect to $r$. (These asymptotics can be proved using Jost solutions, without referring to the theory of Bessel functions.) Using $-\Delta \varphi=$ $\varphi$ we find

$$
-\Delta \psi=\psi-w^{\prime}\left(2 \varphi^{\prime}+(\nu-1) r^{-1} \varphi\right)-\varphi w^{\prime \prime}
$$

with $(\cdot)^{\prime}=\partial / \partial r$. Therefore, the eigenvalue equation for $\psi$ will be satisfied if we set

$$
V:=\frac{w^{\prime}}{w} \frac{2 \varphi^{\prime}+(\nu-1) r^{-1} \varphi}{\varphi}+\frac{w^{\prime \prime}}{w} .
$$

As usual, we want that $w^{\prime}$ vanishes where $\varphi$ vanishes and therefore we define

$$
g(r):=\int_{0}^{r} \varphi(s)^{2} s^{\nu-1} d s=\int_{0}^{r} J_{(\nu-2) / 2}(s)^{2} s d s
$$

The asymptotics (2.2) show that

$$
\lim _{r \rightarrow \infty} r^{-1} g(r)=\pi^{-1}
$$

We now define

$$
w_{n}(r):=\left(n^{2}+g(r)^{2}\right)^{-\alpha}
$$

and we define $V_{n}$ with $w_{n}$ in place of $w$. As in the previous construction, the parameter $n$ need not be an integer, but we will use later that $n \geq 1$. Finally, we will choose $\alpha>\nu / 4$, which by (2.3) will guarantee that $\psi \in L^{2}\left(\mathbb{R}^{\nu}\right)$. As before we do not keep track of how our estimates depend on $\alpha$.

A quick computation shows that

$$
V_{n}=\frac{4 \alpha(\alpha+1)}{m_{n}^{2}} g^{2} g^{\prime 2}-\frac{2 \alpha}{m_{n}}\left(g^{\prime 2}+g g^{\prime \prime}\right)-\frac{2 \alpha}{m_{n}} g g^{\prime} \frac{2 \varphi^{\prime}+(\nu-1) r^{-1} \varphi}{\varphi}
$$

with $m_{n}(r):=n^{2}+g(r)^{2}$. We claim that we can bound

$$
\left|V_{n}\right| \leq C\left(m_{n}^{-1 / 2}+m_{n}^{-1}\right)
$$

with $C$ independent of $n$. Once this is shown we can use $n \geq 1$ to bound $m_{n}^{-1} \leq$ $n^{-1} m_{n}^{-1 / 2} \leq m_{n}^{-1 / 2}$ and obtain $\left|V_{n}\right| \leq C m_{n}^{-1 / 2}$ which, in view of (2.3), is equivalent to the bound stated in the theorem. Clearly this bound will imply $\left\|V_{n}\right\|_{L^{p}} \rightarrow 0$ if $p>\nu$.

Thus, it remains to prove (2.5). Using (2.1) and (2.2) we obtain $g \leq m_{n}^{1 / 2}$ and $\left|g^{\prime}\right|,\left|g^{\prime \prime}\right| \leq C$, which allows us to bound the first two terms on the right side of (2.4) by $C\left(m_{n}^{-1 / 2}+m_{n}^{-1}\right)$. In order to bound the last term, we use $g^{\prime}=\varphi^{2} r^{\nu-1}$, so

$$
g^{\prime} \frac{2 \varphi^{\prime}+(\nu-1) r^{-1} \varphi}{\varphi}=r^{\nu-1} \varphi\left(2 \varphi^{\prime}+(\nu-1) r^{-1} \varphi\right)=\left(r^{\nu-1} \varphi^{2}\right)^{\prime}
$$

Using again (2.1) and (2.2) we obtain $\left|\left(r^{\nu-1} \varphi^{2}\right)^{\prime}\right| \leq C$, and therefore also the last term on the right side of (2.4) is bounded by $C m_{n}^{-1 / 2}$. This completes the proof of (2.5) and of the theorem. 


\section{BOUndS FOR $0 \leq \gamma \leq 1 / 2$}

In this section we review the proofs in [8] and show that these bounds are also valid for positive eigenvalues. Moreover, we shall prove bounds for potentials which belong to spaces of the form $L^{\gamma_{1}+\nu / 2}+L^{\gamma_{2}+\nu / 2}$.

Since we will use a similar argument later in Section 4 we formulate the general principle in abstract terms.

Proposition 3.1. Let $X$ be a separable complex Banach space of functions on $\mathbb{R}^{\nu}$ such that $L^{2}\left(\mathbb{R}^{\nu}\right) \cap X$ is dense in $X$ and such that the duality pairing $X^{*} \times X \rightarrow \mathbb{C}$ extends the inner product in $L^{2}\left(\mathbb{R}^{\nu}\right)$. Assume that

$$
\left\|(-\Delta-z)^{-1}\right\|_{X \rightarrow X^{*}} \leq N(z),
$$

where $N(z)$ is finite for $z \in \mathbb{C} \backslash[0, \infty)$ and continuous up to $[0, \infty) \backslash I$ for some set $I \subset[0, \infty)$. Assume that multiplication by $V: \mathbb{R}^{\nu} \rightarrow \mathbb{C}$ is a bounded operator from $X$ to $X^{*}$. Then, if $E \in \mathbb{C} \backslash I$ is an eigenvalue of $-\Delta+V$ in $L^{2}\left(\mathbb{R}^{\nu}\right)$ with an eigenfunction in $X^{*}$, then

$$
1 \leq N(E)\|V\|_{X^{*} \rightarrow X}
$$

Proof. We give the proof only for $E \in[0, \infty) \backslash I$, the case $E \in \mathbb{C} \backslash[0, \infty)$ being similar (and easier). We denote the eigenfunction by $\psi$ and observe that, since $\psi \in X^{*}$ and since multiplication by $V$ is bounded from $X^{*}$ to $X$,

$$
\|V \psi\|_{X} \leq\|V\|_{X^{*} \rightarrow X}\|\psi\|_{X *}
$$

so $V \psi \in X$. Since $(-\Delta-E-i \varepsilon)^{-1}$ is bounded from $X$ to $X^{*}$ and since, by the eigenvalue equation,

$$
\psi_{\varepsilon}:=(-\Delta-E-i \varepsilon)^{-1}(-\Delta-E) \psi=-(-\Delta-E-i \varepsilon)^{-1}(V \psi),
$$

we infer that $\psi_{\varepsilon} \in X^{*}$ and

$$
\left\|\psi_{\varepsilon}\right\|_{X^{*}} \leq N(E+i \varepsilon)\|V \psi\|_{X}
$$

Since $N(E+i \varepsilon) \rightarrow N(E)$ as $\varepsilon \rightarrow 0$, we see that the $\psi_{\varepsilon}$ are uniformly bounded in $X^{*}$ and so they have a limit point in the weak-* topology of $X^{*}$. On the other hand, by dominated convergence in Fourier space, one easily verifies that $\psi_{\varepsilon} \rightarrow \psi$ strongly (and hence also weakly) in $L^{2}\left(\mathbb{R}^{\nu}\right)$. Since $L^{2}\left(\mathbb{R}^{\nu}\right) \cap X$ is dense in $X$ and since the duality pairing $X^{*} \times X \rightarrow \mathbb{C}$ extends the inner product in $L^{2}\left(\mathbb{R}^{\nu}\right)$, we infer that the limit point in the weak-* topology of $X^{*}$ is unique and given by $\psi$. Moreover, by lower semi-continuity of the norm,

$$
\|\psi\|_{X^{*}} \leq \liminf _{\varepsilon \rightarrow 0}\left\|\psi_{\varepsilon}\right\|_{X^{*}} \leq \liminf _{\varepsilon \rightarrow 0} N(E+i \varepsilon)\|V \psi\|_{X}=N(E)\|V \psi\|_{X}
$$

This, together with the bound (3.2), implies the bound in the proposition.

Our first application of the abstract principle yields the following theorem, which extends the bound of [8] to positive eigenvalues. 
Theorem 3.2. Let $\nu \geq 2,0<\gamma \leq 1 / 2$ and $V \in L^{\gamma+\nu / 2}\left(\mathbb{R}^{\nu}\right)$. Then any eigenvalue $E$ of $-\Delta+V$ in $L^{2}\left(\mathbb{R}^{\nu}\right)$ satisfies

$$
|E|^{\gamma} \leq C_{\gamma, \nu} \int_{\mathbb{R}^{\nu}}|V|^{\gamma+\nu / 2} d x
$$

with $C_{\gamma, \nu}$ independent of $V$. Moreover, if $\nu \geq 3$ and

$$
\int_{\mathbb{R}^{\nu}}|V|^{\nu / 2} d x<C_{\nu}
$$

then $-\Delta+V$ in $L^{2}\left(\mathbb{R}^{\nu}\right)$ has no eigenvalue.

Proof. We apply Proposition 3.1 with $X=L^{p}\left(\mathbb{R}^{\nu}\right)$, where $p$ is defined by $p /(2-p)=$ $\gamma+\nu / 2$, so that the assumptions on $\gamma$ become $2 \nu /(\nu+2)<p \leq 2(\nu+1) /(\nu+3)$. Since $-\Delta+V$ is defined via $m$-sectorial forms, we know a-priori that an eigenfunction satisfies $\psi \in H^{1}\left(\mathbb{R}^{\nu}\right)$ and so, by Sobolev embedding theorems, $\psi \in L^{p^{\prime}}\left(\mathbb{R}^{\nu}\right)=X^{*}$. Note also that, by Hölder's inequality,

$$
\|V\|_{X^{*} \rightarrow X}=\|V\|_{p /(2-p)}
$$

According to the Kenig-Ruiz-Sogge bound (1.2) assumption (3.1) is satisfied with $N(z)=C|z|^{-\nu / 2+\nu / p-1}$ and $I=\{0\}$. Therefore the claimed bound follows from Proposition 3.1. The second part of the theorem is proved similarly, taking $\gamma=0$, $I=\emptyset$ and noting that for $\nu \geq 3$ the bound (1.2) holds also for $p=2 \nu /(\nu+2)$. This completes the proof.

Remark 3.3. In a similar spirit we note that if $\nu=1$ and $V \in L^{1}(\mathbb{R}$ ) (possibly complex-valued), then $-d^{2} / d x^{2}+V(x)$ in $L^{2}(\mathbb{R})$ has no positive eigenvalue. Thus the restriction that the bound $|E|^{1 / 2} \leq(1 / 2)\|V\|_{1}$ holds only for eigenvalues $E \in$ $\mathbb{C} \backslash(0, \infty)$, which appears frequently in the literature, is unnecessary. (The absence of positive eigenvalues follows from standard Jost function techniques which show that for $k>0$ the equation $-\psi^{\prime \prime}+V \psi=k^{2} \psi$ has two solutions $\psi_{+}$and $\psi_{-}$with $\psi_{ \pm}(x) \sim e^{ \pm i k x}$ as $x \rightarrow \infty$, so no solution of this equation is square integrable. These arguments go back at least to Titchmarsh [33].)

Proposition 3.4. Let $V_{1} \in L^{\gamma_{1}+\nu / 2}\left(\mathbb{R}^{\nu}\right), V_{2} \in L^{\gamma_{2}+\nu / 2}\left(\mathbb{R}^{\nu}\right)$, where $0<\gamma_{1}<\gamma_{2} \leq 1 / 2$ if $\nu=2$ and $0 \leq \gamma_{1}<\gamma_{2} \leq 1 / 2$ if $\nu \geq 3$. Then any eigenvalue $E \in \mathbb{C} \backslash\{0\}$ of $-\Delta+V_{1}+V_{2}$ in $L^{2}\left(\mathbb{R}^{\nu}\right)$ satisfies

$$
|E|^{-\gamma_{1}} \int_{\mathbb{R}^{\nu}}\left|V_{1}\right|^{\gamma_{1}+\nu / 2} d x+|E|^{-\gamma_{2}} \int_{\mathbb{R}^{\nu}}|V|^{\gamma_{2}+\nu / 2} d x \geq c_{\gamma_{1}, \gamma_{2}, \nu}>0
$$

Proof. Again we prove this only for positive eigenvalues, the other case being simpler. Let $\psi$ be the eigenfunction and let $\varepsilon>0$ be a small parameter. We denote $S_{\varepsilon}:=$ $|-\Delta-E-i \varepsilon|(-\Delta-E-i \varepsilon)^{-1}$ and $\varphi_{\varepsilon}:=|-\Delta-E-i \varepsilon|^{1 / 2} \psi$, where $\psi$ is the eigenfunction. Since $\psi \in H^{1}\left(\mathbb{R}^{\nu}\right), \varphi_{\varepsilon} \in L^{2}\left(\mathbb{R}^{\nu}\right)$. We can write the eigenvalue equation in the form

$$
S_{\varepsilon}|-\Delta-E-i \varepsilon|^{-1 / 2} V|-\Delta-E-i \varepsilon|^{-1 / 2} \varphi_{\varepsilon}=-\frac{-\Delta-E}{-\Delta-E-i \varepsilon} \varphi_{\varepsilon} .
$$


Therefore,

$$
\begin{aligned}
\left\|\frac{-\Delta-E}{-\Delta-E-i \varepsilon} \varphi_{\varepsilon}\right\| & =\left\|S_{\varepsilon}|-\Delta-E-i \varepsilon|^{-1 / 2} V|-\Delta-E-i \varepsilon|^{-1 / 2} \varphi_{\varepsilon}\right\| \\
\leq & \left(|| S_{\varepsilon}|-\Delta-E-i \varepsilon|^{-1 / 2} V_{1}|-\Delta-E-i \varepsilon|^{-1 / 2} \|\right. \\
& \left.\quad+|| S_{\varepsilon}|-\Delta-E-i \varepsilon|^{-1 / 2} V_{2}|-\Delta-E-i \varepsilon|^{-1 / 2} \|\right)\left\|\varphi_{\varepsilon}\right\| .
\end{aligned}
$$

Since the operator norm of $A B$ equals that of $B A$, we have

$$
|| S_{\varepsilon}|-\Delta-E-i \varepsilon|^{-1 / 2} V_{j}|-\Delta-E-i \varepsilon|^{-1 / 2}\|=\|\left(\operatorname{sgn} V_{j}\right)\left|V_{j}\right|^{1 / 2}(-\Delta-E-i \varepsilon)^{-1}\left|V_{j}\right|^{1 / 2} \|
$$

and, as in [8], the Kenig-Ruiz-Sogge bound (1.2) implies that

$$
\left\|\left(\operatorname{sgn} V_{j}\right)\left|V_{j}\right|^{1 / 2}(-\Delta-E-i \varepsilon)^{-1}\left|V_{j}\right|^{1 / 2}\right\| \leq C\left(|E|^{2}+\varepsilon^{2}\right)^{-\gamma_{j} /\left(2 \gamma_{j}+\nu\right)}\left\|V_{j}\right\|_{\gamma_{j}+\nu / 2} .
$$

Inserting this into (3.3) we obtain

$$
\begin{aligned}
\left\|\frac{-\Delta-E}{-\Delta-E-i \varepsilon} \varphi_{\varepsilon}\right\| \leq C & \left(\left(|E|^{2}+\varepsilon^{2}\right)^{-\gamma_{1} /\left(2 \gamma_{1}+\nu\right)}\left\|V_{1}\right\|_{\gamma_{1}+\nu / 2}\right. \\
& \left.+\left(|E|^{2}+\varepsilon^{2}\right)^{-\gamma_{2} /\left(2 \gamma_{2}+\nu\right)}\left\|V_{2}\right\|_{\gamma_{2}+\nu / 2}\right)\left\|\varphi_{\varepsilon}\right\| .
\end{aligned}
$$

Finally, we observe that $\left\|\varphi_{\varepsilon}\right\| \leq\|\varphi\|<\infty$ and that $\frac{-\Delta-E}{-\Delta-E-i \varepsilon} \varphi_{\varepsilon} \rightarrow \varphi$ in $L^{2}\left(\mathbb{R}^{\nu}\right)$ (by dominated convergence in Fourier space. Thus, as $\varepsilon \rightarrow 0$, we obtain the claimed bound in the theorem.

\section{BOUnDS FOR $1 / 2<\gamma<\nu / 2$}

4.1. Eigenvalue bounds. In this section we show that (1.1) holds for $1 / 2<\gamma<$ $\nu / 2$ if $V$ is radial and, more generally, if for every $r>0, V(r \omega)$ is replaced by $\operatorname{ess}^{-\sup _{\omega \in \mathbb{S}^{\nu-1}}}|V(r \omega)|$. The precise statement is

Theorem 4.1. Let $\nu \geq 2$ and $1 / 2<\gamma<\nu / 2$. Then

$$
|E|^{\gamma} \leq C_{\gamma, \nu} \int_{0}^{\infty}\|V(r \cdot)\|_{L^{\infty}\left(\mathbb{S}^{\nu-1}\right)}^{\gamma+\nu / 2} r^{\nu-1} d r .
$$

At the endpoint $\gamma=\nu / 2$ we have the following bound

Theorem 4.2. Let $\nu \geq 2$. Then

$$
|E|^{\nu / 2} \leq C_{\nu}\left(\int_{0}^{\infty}\left|\left\{r>0: \operatorname{ess}^{-\sup _{\omega \in \mathbb{S}^{\nu-1}}}|V(r \omega)|>\tau\right\}\right|_{\nu}^{1 / \nu} d \tau\right)^{\nu}
$$

where $|\cdot|_{\nu}$ denotes the measure $\left|\mathbb{S}^{\nu-1}\right| r^{\nu-1} d r$ on $(0, \infty)$

Note that the integral on the right side in the theorem is the norm in the Lorentz space $L^{\nu, 1}\left(\mathbb{R}_{+}, r^{\nu-1} d r ; L^{\infty}\left(\mathbb{S}^{\nu-1}\right)\right)$.

We will deduce Theorems 4.1 and 4.2 from the following two resolvent bounds. The first one will imply Theorem 4.1 . 
Theorem 4.3. Let $\nu \geq 2$ and $2(\nu+1) /(\nu+3)<p<2 \nu /(\nu+1)$. Then for all $f \in L^{p}\left(\mathbb{R}_{+}, r^{\nu-1} d r ; L^{2}\left(\mathbb{S}^{\nu-1}\right)\right)$ and $z \in \mathbb{C} \backslash[0, \infty)$,

$$
\begin{gathered}
\left(\int_{0}^{\infty}\left(\int_{\mathbb{S}^{\nu-1}}\left|\left((-\Delta-z)^{-1} f\right)(r \omega)\right|^{2} d \omega\right)^{p^{\prime} / 2} r^{\nu-1} d r\right)^{1 / p^{\prime}} \\
\quad \leq C_{p, \nu}|z|^{-\nu / 2+\nu / p-1}\left(\int_{0}^{\infty}\left(\int_{\mathbb{S}^{\nu-1}}|f(r \omega)|^{2} d \omega\right)^{p / 2} r^{\nu-1} d r\right)^{1 / p}
\end{gathered}
$$

As explained in the introduction, we think of Theorem 4.3 as the analogue of the uniform Sobolev bounds by Kenig-Ruiz-Sogge [16] which correspond to the range $2 \nu /(\nu+2)<p \leq 2(\nu+1) /(\nu+3)$, see (1.2). Since uniform resolvent bounds imply Fourier restriction bounds (since $(-\Delta-\lambda-i \varepsilon)^{-1}-(-\Delta-\lambda+i \varepsilon)^{-1} \rightarrow 2 \pi i \delta(-\Delta-\lambda)$ as $\varepsilon \rightarrow 0+$ ), the Knapp counterexample [32] shows that (1.2) cannot hold for larger values of $p$. However, as we show, larger values of $p$ can be achieved by considering mixed norm spaces. The use of mixed norm spaces in the context of Fourier restriction bounds seems to have first appeared in Vega [34], who proved the corresponding restriction inequality in the range $2(\nu+1) /(\nu+3)<p<2 \nu /(\nu+1)$ in dimensions $\nu \geq 3$; see also [13] where $\nu=2$ is included as well. Our resolvent bound seems to be new, although our arguments follow closely those of Barcelo-Ruiz-Vega [2], and our assumption $p<2 \nu /(\nu+1)$ is optimal, since the results of [13] show that the corresponding Fourier restriction bound does not hold for $p \geq 2 \nu /(\nu+1)$.

The following bound will imply Theorem 4.2. As we will see, it is a rather straightforward consequence of the main result of [2].

Theorem 4.4. Let $\nu \geq 2$ and let $V$ be a non-negative, measurable function with

$$
\|V\|_{L^{\nu, 1}\left(\mathbb{R}_{+}, r^{\nu-1} d r ; L^{\infty}\left(\mathbb{S}^{\nu-1}\right)\right)}=\int_{0}^{\infty}\left|\left\{r>0: \operatorname{ess-}_{\sup _{\omega \in \mathbb{S}}^{\nu-1}}|V(r \omega)|>\tau\right\}\right|_{\nu}^{1 / \nu} d \tau<\infty .
$$

Then, for all $f \in L^{2}\left(\mathbb{R}^{\nu}, V^{-1} d x\right) \cap L^{2}\left(\mathbb{R}^{\nu}\right)$ and $z \in \mathbb{C} \backslash[0, \infty)$,

$$
\int_{\mathbb{R}^{\nu}}\left|(-\Delta-z)^{-1} f\right|^{2} V d x \leq C|z|^{-1}\|V\|_{L^{\nu, 1}\left(\mathbb{R}_{+}, r^{\nu-1} d r ; L^{\infty}\left(\mathbb{S}^{\nu-1}\right)\right)}^{2} \int_{\mathbb{R}^{\nu}}|f|^{2} V^{-1} d x .
$$

Theorem 4.1 follows from Theorem 4.3 by Proposition 3.1 with the choice $X=$ $L^{p}\left(\mathbb{R}_{+}, r^{\nu-1} d r ; L^{2}\left(\mathbb{S}^{\nu-1}\right)\right)$ in the same way as Theorem 3.2 was derived from (1.2). Similarly, Theorem 4.2 follows from Theorem 4.4 by Proposition 3.1, here we set $X=L^{2}\left(w^{-1}\right)$ where $w=\max \{|V|, \delta G\}$, where $G$ is a strictly positive function in $L^{\nu, 1}\left(\mathbb{R}_{+}, r^{\nu-1} d r ; L^{\infty}\left(\mathbb{S}^{\nu-1}\right)\right.$ ) (for instance, a Gaussian) and $\delta>0$ is a small parameter. Having $\delta>0$ implies that $L^{2} \cap L^{2}\left(w^{-1}\right)$ is dense in $L^{2}\left(w^{-1}\right)$. Moreover, one easily verifies that

$$
\|V\|_{L^{2}(w) \rightarrow L^{2}\left(w^{-1}\right)} \leq 1
$$

so Proposition 3.1 yields

$$
1 \leq C|z|^{-1}\|\max \{|V|, \delta G\}\|_{L^{\nu, 1}\left(\mathbb{R}_{+}, r^{\nu-1} d r ; L^{\infty}\left(\mathbb{S}^{\nu-1}\right)\right)}^{2}
$$


and as $\delta \rightarrow 0$ we obtain the claimed bound.

Thus, it remains to prove Theorems 4.3 and 4.4 .

4.2. Proof of Theorem 4.3. It is well known that on spherical harmonics of degree $l \in \mathbb{N}_{0}$ the operator $-\Delta$ acts as

$$
h_{l}:=-\partial_{r}^{2}-(\nu-1) r^{-1} \partial_{r}+l(l+\nu-2) r^{-2} .
$$

This operator, with an appropriate boundary condition at the origin (coming from the decomposition into spherical harmonics), is self-adjoint in $L^{2}\left(\mathbb{R}_{+}, r^{\nu-1} d r\right)$. It is well-known that the boundary values of the resolvent $\left(h_{l}-\lambda-i 0\right)^{-1}$ exist in suitably weighted spaces. The following proposition shows that these boundary values are bounded operators from $L^{p}\left(\mathbb{R}_{+}, r^{\nu-1} d r\right)$ to $L^{p^{\prime}}\left(\mathbb{R}_{+}, r^{\nu-1} d r\right)$. The key observation is that their norms are bounded uniformly in $l \in \mathbb{N}_{0}$.

Proposition 4.5. For any $\nu \geq 2$ and $2 \nu /(\nu+2)<p<2 \nu /(\nu+1)$,

$$
\sup _{l \in \mathbb{N}_{0}}\left\|\left(h_{l}-1-i 0\right)^{-1}\right\|_{L^{p}\left(\mathbb{R}_{+}, r^{\nu-1}\right) \rightarrow L^{p^{\prime}}\left(\mathbb{R}_{+}, r^{\nu-1}\right)}<\infty .
$$

To prove this proposition we use the following simple criterion for the boundedness of an integral operator from $L^{p}$ to $L^{p^{\prime}}$.

Lemma 4.6. Let $X$ and $Y$ be measure spaces and $k \in L^{p^{\prime}}(X \times Y)$ for some $1 \leq p \leq 2$. Then $(k f)(y)=\int_{X} k(x, y) f(x) d x$ defines a bounded operator from $L^{p}(X)$ to $L^{p^{\prime}}(Y)$ with

$$
\|k\|_{L^{p}(X) \rightarrow L^{p^{\prime}}(Y)} \leq\|k\|_{L^{p^{\prime}(X \times Y)}} .
$$

Proof of Lemma 4.6. By Minkowski's and Hölder's inequality

$$
\begin{aligned}
\|k f\|_{p^{\prime}}^{p^{\prime}} & =\int_{Y}\left|\int_{X} k(x, y) f(x) d x\right|^{p^{\prime}} d y \\
& \leq\left(\int_{X}\left(\int_{Y}|k(x, y)|^{p^{\prime}} d y\right)^{1 / p^{\prime}}|f(x)| d x\right)^{p^{\prime}} \\
& \leq\left(\int_{X} \int_{Y}|k(x, y)|^{p^{\prime}} d y d x\right)\left(\int_{X}|f(x)|^{p} d x\right)^{p^{\prime} / p},
\end{aligned}
$$

which yields the claimed inequality.

Modulo a technical result about Bessel functions (Proposition A.1), which we prove in the appendix, we now give the

Proof of Proposition 4.5. According to Sturm-Liouville theory $\left(h_{l}-1-i 0\right)^{-1}$ is an integral operator with integral kernel

$$
\left(h_{l}-1-i 0\right)^{-1}\left(r, r^{\prime}\right)=\left(r r^{\prime}\right)^{-(\nu-2) / 2} J_{\mu_{l}}\left(\min \left\{r, r^{\prime}\right\}\right) H_{\mu_{l}}^{(1)}\left(\max \left\{r, r^{\prime}\right\}\right),
$$


where $J_{\mu_{l}}$ and $H_{\mu_{l}}^{(1)}$ are Bessel and Hankel functions, respectively, and where $\mu_{l}=$ $l+(\nu-2) / 2$. Thus, by Lemma 4.6,

$$
\begin{aligned}
& \left\|\left(h_{l}-1-i 0\right)^{-1}\right\|_{L^{p}\left(\mathbb{R}_{+}, r^{\nu-1}\right) \rightarrow L^{p^{\prime}}\left(\mathbb{R}_{+}, r^{\nu-1}\right)}^{p^{\prime}} \\
& \quad \leq 2 \int_{0}^{\infty} \int_{r}^{\infty}\left|J_{\mu_{l}}(r)\right|^{p^{\prime}}\left|H_{\mu_{l}}\left(r^{\prime}\right)\right|^{p^{\prime}}\left(r r^{\prime}\right)^{-p^{\prime}(\nu-2) / 2+\nu-1} d r^{\prime} d r .
\end{aligned}
$$

The fact that the right side is finite and uniformly bounded in $l$ follows from Proposition A.1 in the appendix with $q=p^{\prime}$. This completes the proof of the proposition.

In order to deduce Theorem 4.3 from Proposition 4.5 we need the following general result.

Lemma 4.7. Let $X$ and $Y$ be measure spaces and $1 \leq p \leq 2$. Let $\left(K_{j}\right)$ be a sequence of bounded operators from $L^{p}(X)$ to $L^{p^{\prime}}(Y)$. Let $\mathcal{H}$ be a separable Hilbert space with an orthonormal basis $\left(e_{j}\right)$ and define a linear operator $K$ by

$$
K\left(f \otimes e_{j}\right)=\left(K_{j} f\right) \otimes e_{j} \quad \text { for all } f \in L^{p}(X) \text { and all } j .
$$

Then $K$ is bounded from $L^{p}(X, \mathcal{H})$ to $L^{p^{\prime}}(Y, \mathcal{H})$ with

$$
\|K\|_{L^{p}(X, \mathcal{H}) \rightarrow L^{p^{\prime}}(Y, \mathcal{H})}=\sup _{j}\left\|K_{j}\right\|_{L^{p}(X) \rightarrow L^{p^{\prime}}(Y)} .
$$

Proof of Lemma 4.7. Since

$$
\begin{aligned}
\left\|K\left(f \otimes e_{j}\right)\right\|_{L^{p^{\prime}}(Y, \mathcal{H})} & =\left\|K_{j} f\right\|_{L^{p^{\prime}(Y)}} \leq\left\|K_{j}\right\|_{L^{p}(X) \rightarrow L^{p^{\prime}}(Y)}\|f\|_{L^{p}(X)} \\
& =\left\|K_{j}\right\|_{L^{p}(X) \rightarrow L^{p^{\prime}}(Y)}\left\|f \otimes e_{j}\right\|_{L^{p}(Y, \mathcal{H})},
\end{aligned}
$$

we have $\|K\| \leq \sup \left\|K_{j}\right\|$ (with obvious indices). To prove the opposite bound we write $F=\sum f_{j} \otimes e_{j}$, so that

$$
\|K F\|_{L^{p^{\prime}(Y, \mathcal{H})}}^{p^{\prime}}=\int_{Y}\left(\sum\left|\left(K_{j} f_{j}\right)(y)\right|^{2}\right)^{p^{\prime} / 2} d y .
$$

Since $p^{\prime} \geq 2$ we can bound this from above using Minkowski's inequality by

$$
\left(\sum\left(\int_{Y}\left|\left(K_{j} f_{j}\right)(y)\right|^{p^{\prime}} d y\right)^{2 / p^{\prime}}\right)^{p^{\prime} / 2}
$$

which in turn is bounded from above by

$$
\left(\sum\left\|K_{j}\right\|^{2}\left(\int_{X}\left|f_{j}(x)\right|^{p} d x\right)^{2 / p}\right)^{p^{\prime} / 2} \leq\left(\sup \left\|K_{j}\right\|\right)^{p^{\prime}}\left(\sum\left(\int_{X}\left|f_{j}(x)\right|^{p} d x\right)^{2 / p}\right)^{p^{\prime} / 2} \text {. }
$$

Once again by Minkowski's inequality, using the fact that $p \leq 2$,

$$
\sum\left(\int_{X}\left|f_{j}(x)\right|^{p} d x\right)^{2 / p} \leq\left(\int_{X}\left(\sum\left|f_{j}(x)\right|^{2}\right)^{p / 2} d x\right)^{2 / p}=\|F\|_{L^{p}(X, \mathcal{H})}^{2} .
$$

This proves that $\|K F\|_{L^{p^{\prime}}(Y, \mathcal{H})} \leq\left(\sup \left\|K_{j}\right\|\right)\|F\|_{L^{p}(X, \mathcal{H})}$, as claimed.

We are finally in position to give the 
Proof of Theorem 4.3. Let $\nu \geq 2$ and $2(\nu+1) /(\nu+3)<p<2 \nu /(\nu+1)$. (In fact, the proof works also for $2 \nu /(\nu+2)<p \leq 2(\nu+1) /(\nu+3)$, but the inequality we obtain in that case is weaker than (1.2).) We begin with a well-known argument reducing the proof to the case $z=1$. For $f, g \in C_{0}^{\infty}\left(\mathbb{R}^{\nu}\right)$,

$$
z \mapsto z^{\nu / 2-\nu / p+1}\left(g,(-\Delta-z)^{-1} f\right)
$$

is an analytic function in $\{\operatorname{Im} z>0\}$, continuous up to the boundary, and satisfying

$$
|z|^{\nu / 2-\nu / p+1}\left|\left(g,(-\Delta-z)^{-1} f\right)\right| \leq C_{r, \nu}|z|^{\alpha}\|f\|_{r}\|g\|_{r}
$$

for every $2 \nu /(\nu+2)<r \leq 2(\nu+1) /(\nu+3)$ and a certain $\alpha$ depending on $r$. This follows from the Kenig-Ruiz-Sogge bound (1.2). Thus, by the Phragmén-Lindelöf principle,

$$
\sup _{\operatorname{Im} z>0}|z|^{\nu / 2-\nu / p+1}\left|\left(g,(-\Delta-z)^{-1} f\right)\right|=\sup _{\lambda \in \mathbb{R}}|\lambda|^{\nu / 2-\nu / p+1}\left|\left(g,(-\Delta-\lambda-i 0)^{-1} f\right)\right| .
$$

If we can show that the right side is bounded by $C_{p, \nu}\|f\|_{L^{p}\left(L^{2}\right)}\|g\|_{L^{p}\left(L^{2}\right)}$ (with the abbreviation $\left.L^{p}\left(L^{2}\right)=L^{p}\left(\mathbb{R}_{+}, r^{\nu-1} d r ; L^{2}\left(\mathbb{S}^{\nu-1}\right)\right)\right)$, then, by density, the bound will be valid for any $f, g \in L^{p}\left(L^{2}\right)$. Moreover, since

$$
\left(g,(-\Delta-\bar{z})^{-1} f\right)=\left((-\Delta-z)^{-1} g, f\right)=\overline{\left(f,(-\Delta-z)^{-1} g\right)},
$$

we will have shown the bound claimed in the theorem.

By scaling it suffices to prove the bound

$$
|\lambda|^{\nu / 2-\nu / p+1}\left|\left(g,(-\Delta-\lambda-i 0)^{-1} f\right)\right| \leq C_{p, \nu}\|f\|_{L^{p}\left(L^{2}\right)}\|g\|_{L^{p}\left(L^{2}\right)}
$$

for $\lambda= \pm 1$ only. We begin with $\lambda=-1$. Since $(-\Delta+1)^{-1}$ is convolution with a function in $L^{q}$ for any $q<\nu /(\nu-2)$, Young's inequality yields

$$
\left|\left(g,(-\Delta-\lambda-i 0)^{-1} f\right)\right| \leq C_{p, \nu}^{\prime}\|f\|_{L^{p}}\|g\|_{L^{p}}
$$

for any $p>2 \nu /(\nu+2)$. Since

$$
\|f\|_{L^{p}} \leq\left|\mathbb{S}^{\nu-1}\right|^{(2-p) / 2 p}\|f\|_{L^{p}\left(L^{2}\right)}
$$

for $p \leq 2$, this bound for $\lambda=-1$ is stronger than what we shall prove for $\lambda=1$.

Therefore we have reduced the proof to showing (4.1) for $2(\nu+1) /(\nu+3)<p<$ $2 \nu /(\nu+1)$ and $\lambda=1$. This is the same as

$$
\left\|(-\Delta-1-i 0)^{-1} f\right\|_{L^{p^{\prime}\left(L^{2}\right)}} \leq C_{p, \nu}\|f\|_{L^{p}\left(L^{2}\right)} .
$$

To do so, we expand $f$ with respect to spherical harmonics $\left(Y_{l, m}\right)$, with $l \in \mathbb{N}_{0}$ and $m$ running through a certain index set of cardinality depending on $l$,

$$
f(x)=\sum_{l, m} f_{l, m}(|x|) Y_{l, m}(x /|x|),
$$

so that

$$
\int_{0}^{\infty}\left(\int_{\mathbb{S}^{\nu-1}}|f(r \omega)|^{2} d \omega\right)^{p / 2} r^{\nu-1} d r=\int_{0}^{\infty}\left(\sum_{l, m}\left|f_{l, m}(r)\right|^{2}\right)^{p / 2} r^{\nu-1} d r
$$


Separation of variables shows that

$$
\left((-\Delta-1-i 0)^{-1} f\right)(x)=\sum_{l m}\left(\left(h_{l}-1-i 0\right)^{-1} f_{l m}\right)(|x|) Y_{l m}(x /|x|),
$$

where $h_{l}$ was defined at the beginning of this subsection. By Lemma 4.7 we have

$$
\left\|(-\Delta-1-i 0)^{-1}\right\|_{L^{p}\left(L^{2}\right) \rightarrow L^{p^{\prime}}\left(L^{2}\right)}=\sup _{l \in \mathbb{N}_{0}}\left\|\left(h_{l}-1+i 0\right)^{-1}\right\|_{L^{p} \rightarrow L^{p^{\prime}}} .
$$

The right hand side is finite by Proposition 4.3. This completes the proof of the theorem.

4.3. Proof of Theorem 4.4. We shall deduce Theorem 4.4 from the following theorem of Barcelo, Ruiz and Vega [2]. They introduce the following norm,

$$
\|V\|_{M T}=\sup _{R>0} \int_{R}^{\infty} \frac{\operatorname{ess}_{R} \sup _{\omega \in \mathbb{S}^{\nu-1}}|V(r \omega)| r}{\left(r^{2}-R^{2}\right)^{1 / 2}} d r<\infty .
$$

Theorem 4.8. Let $\nu \geq 2$ and let $V$ be a non-negative, measurable function with $\|V\|_{M T}<\infty$. Then, for all $f \in L^{2}\left(\mathbb{R}^{\nu}, V^{-1} d x\right) \cap L^{2}\left(\mathbb{R}^{\nu}\right)$ and $z \in \mathbb{C} \backslash[0, \infty)$,

$$
\int_{\mathbb{R}^{\nu}}\left|(-\Delta-z)^{-1} f\right|^{2} V d x \leq C|z|^{-1}\|V\|_{M T}^{2} \int_{\mathbb{R}^{\nu}}|f|^{2} V^{-1} d x .
$$

Barcelo, Ruiz and Vega call $\|V\|_{M T}<\infty$ the 'radial Mizohata-Takeuchi' condition, thus the subscript 'MT'. They show that for radial $V$ this condition is, in fact, also necessary to have a bound of the form $\|u\|_{L^{2}(V)} \leq C|z|^{-1 / 2}\|(-\Delta-z) u\|_{L^{2}(V)}$.

Proof of Theorem 4.4. By Theorem 4.8 it suffices to show that for any $\nu \geq 2$,

$$
\|V\|_{M T} \leq C_{\nu}\|V\|_{L^{\nu, 1}\left(\mathbb{R}_{+}, r^{\nu-1}, L^{\infty}\left(\mathbb{S}^{\nu-1}\right)\right)} .
$$

Let $\rho_{R}(r):=r^{-\nu+2}\left(r^{2}-R^{2}\right)^{-1 / 2} \chi_{\{r>R\}}$. Then, by Hölder's inequality in Lorentz spaces, with $v(r):=\operatorname{ess-sup}_{\omega \in \mathbb{S}^{\nu-1}}|V(r \omega)|$,

$$
\begin{aligned}
\int_{R}^{\infty} \frac{\operatorname{ess-sup}_{\omega \in \mathbb{S}^{\nu-1}}|V(r \omega)| r}{\left(r^{2}-R^{2}\right)^{1 / 2}} d r & =\int_{0}^{\infty} v(r) \rho_{R}(r) r^{\nu-1} d r \\
& \leq C\|v\|_{L^{\nu, 1}\left(\mathbb{R}_{+}, r^{\nu-1}\right)}\left\|\rho_{R}\right\|_{L^{\nu /(\nu-1), \infty\left(\mathbb{R}_{+}, r^{\nu-1}\right)}} \\
& =C\|V\|_{L^{\nu, 1}\left(\mathbb{R}_{+}, r^{\nu-1}, L^{\infty}\left(\mathbb{S}^{\nu-1}\right)\right)}\left\|\rho_{1}\right\|_{L^{\nu /(\nu-1), \infty}\left(\mathbb{R}_{+}, r^{\nu-1}\right)}
\end{aligned}
$$

where we used that, by scaling, $\left\|\rho_{R}\right\|_{L^{\nu /(\nu-1), \infty\left(\mathbb{R}_{+}, r^{\nu-1}\right)}}=\left\|\rho_{1}\right\|_{L^{\nu /(\nu-1), \infty\left(\mathbb{R}_{+}, r^{\nu-1}\right)}}$. One easily checks that $\rho_{1} \in L^{\nu /(\nu-1), \infty}\left(\mathbb{R}_{+}, r^{\nu-1}\right)$, which, after taking the supremeum over $R>0$, yields (4.2).

The next corollary contains further eigenvalue bounds which are consequences of Theorem 4.8,

Corollary 4.9. Let $E \in \mathbb{C}$ be an eigenvalue of $-\Delta+V$ in $L^{2}\left(\mathbb{R}^{\nu}\right)$. Then

$$
|E|^{1 / 2} \leq C_{\nu}\|V\|_{M T} .
$$


Moreover, for any $p \in(2, \infty]$,

$$
|E|^{1 / 2} \leq C_{p, \nu} \sum_{j \in \mathbb{Z}}\left(\int_{2^{j}}^{2^{j+1}}\|V(r \cdot)\|_{L^{\infty}\left(\mathbb{S}^{\nu-1}\right)}^{p} r^{p-1} d r\right)^{1 / p} .
$$

Clearly, (4.4) for $p=\infty$ means

$$
|E|^{1 / 2} \leq C_{\nu} \sum_{j \in \mathbb{Z}}\left(\sup _{2^{j}<|x|<2^{j+1}}|x||V(x)|\right) .
$$

Since $\sum_{j \in \mathbb{Z}}\left(\sup _{2^{j}<|x|<2^{j+1}}|x|(1+|x|)^{-1-\varepsilon}\right)<\infty$ for $\varepsilon>0$, this bound implies, in particular,

$$
|E|^{1 / 2} \leq C_{\nu, \varepsilon} \operatorname{ess-sup}_{x \in \mathbb{R}^{\nu}}(1+|x|)^{1+\varepsilon}|V(x)|, \quad \varepsilon>0 .
$$

which is the main result of 28 .

Proof. Bound (4.3) follows from Theorem 4.8 by Proposition 3.1 using the arguments after Theorem 4.4. Having proved this, for (4.4) it suffices to prove that

$$
\|V\|_{M T} \leq C_{p, \nu} \sum_{j \in \mathbb{Z}}\left(\int_{2^{j}}^{2^{j+1}}\|V(r \cdot)\|_{L^{\infty}\left(\mathbb{S}^{n u-1}\right)}^{p} r^{p-1} d r\right)^{1 / p} .
$$

This bound is stated in 2] without proof, so we include it for the sake of completeness. We abbreviate $v(r):=\|V(r \cdot)\|_{L^{\infty}\left(\mathbb{S}^{\nu-1}\right)}$. Since $p>2$,

$$
\begin{aligned}
\int_{R}^{2 R 1} \frac{v(r) r}{\left(r^{2}-R^{2}\right)^{1 / 2}} d r & \leq\left(\int_{R}^{2 R} v(r)^{p} r^{p-1} d r\right)^{1 / p}\left(\int_{R}^{2 R}\left(\frac{r}{\sqrt{r^{2}-R^{2}}}\right)^{p^{\prime}} \frac{d r}{r}\right)^{1 / p^{\prime}} \\
& =c_{p}\left(\int_{R}^{2 R} v(r)^{p} r^{p-1} d r\right)^{1 / p} .
\end{aligned}
$$

On the other hand, for $r \geq 2 R, r / \sqrt{r^{2}-R^{2}} \leq 2 / \sqrt{3}$, and therefore

$$
\begin{aligned}
\int_{2 R}^{\infty} \frac{v(r) r}{\left(r^{2}-R^{2}\right)^{1 / 2}} d r & \leq \frac{2}{\sqrt{3}} \sum_{j=1}^{\infty} \int_{2^{j} R}^{2^{j+1} R} v(r) d r \\
& \leq \frac{2}{\sqrt{3}} \sum_{j=1}^{\infty}\left(\int_{2^{j} R}^{2^{j+1} R} v(r)^{p} r^{p-1} d r\right)^{1 / p}\left(\int_{2^{j} R}^{2^{j+1} R} \frac{d r}{r}\right)^{1 / p^{\prime}} \\
& =\frac{2}{\sqrt{3}}(\ln 2)^{1 / p^{\prime}} \sum_{j=1}^{\infty}\left(\int_{2^{j} R}^{2^{j+1} R} v(r)^{p} r^{p-1} d r\right)^{1 / p} .
\end{aligned}
$$

Picking $k \in \mathbb{Z}$ such that $2^{k} \leq R<2^{k+1}$ we easily deduce (4.5). 


\section{Appendix A. Bounds on Bessel functions}

The key ingredient in our proof of Proposition 4.5] was the following result about integrals of Bessel and Hankel functions.

Proposition A.1. Let $\nu \geq 2$ and $2 \nu /(\nu-1)<q<2 \nu /(\nu-2)$. Then

$$
\sup _{\mu \geq 0} \int_{0}^{\infty} \int_{r}^{\infty}\left|J_{\mu}(r)\right|^{q}\left|H_{\mu}^{(1)}\left(r^{\prime}\right)\right|^{q}\left(r r^{\prime}\right)^{-q(\nu-2) / 2+\nu-1} d r d r^{\prime}<\infty .
$$

We emphasize that in this result $\nu$ is not required to be integer and $\mu$ is not required to be a half-integer (although they will be in our application later on).

In this appendix we prove Proposition A.1 using the techniques of [2]. Using WKB analysis, Barcelo, Ruiz and Vega prove the following uniform bounds on Bessel functions. We state their complete result although we will not use its full strength.

Proposition A.2. There is a constant $C>0$ and a constant $\alpha_{0} \in(0,1 / 2)$ such that the following holds for all $\mu \geq 1 / 2$.

(1) For $0<r \leq 1$,

$$
\left|J_{\mu}(r)\right| \leq C \frac{(r / 2)^{\mu}}{\Gamma(\mu+1)}, \quad\left|H_{\mu}^{(1)}(r)\right| \leq C \frac{\Gamma(\mu)}{(r / 2)^{\mu}} .
$$

(2) For $1 \leq r \leq \mu \operatorname{sech} \alpha_{0}$

$$
\left|J_{\mu}(r)\right| \leq C \frac{e^{-\mu \varphi_{\mu}(r)}}{\mu^{1 / 2}}, \quad\left|H_{\mu}^{(1)}(r)\right| \leq C \frac{e^{\mu \varphi_{\mu}(r)}}{\mu^{1 / 2}} .
$$

(3) For $\mu \operatorname{sech} \alpha_{0} \leq r \leq \mu-\mu^{1 / 3}$,

$$
\left|J_{\mu}(r)\right| \leq C \frac{e^{-\mu \varphi_{\mu}(r)}}{\mu^{1 / 4}(\mu-r)^{1 / 4}}, \quad\left|H_{\mu}^{(1)}(r)\right| \leq C \frac{e^{\mu \varphi_{\mu}(r)}}{\mu^{1 / 4}(\mu-r)^{1 / 4}} .
$$

(4) For $\mu-\mu^{1 / 3} \leq r \leq \mu+\mu^{1 / 3}$,

$$
\left|J_{\mu}(r)\right| \leq C \frac{1}{\mu^{1 / 3}}, \quad\left|H_{\mu}^{(1)}(r)\right| \leq C \frac{1}{\mu^{1 / 3}} .
$$

(5) For $r \geq \mu+\mu^{1 / 3}$

$$
\left|J_{\mu}(r)\right| \leq C \frac{1}{r^{1 / 4}(r-\mu)^{1 / 4}}, \quad\left|H_{\mu}^{(1)}(r)\right| \leq C \frac{1}{r^{1 / 4}(r-\mu)^{1 / 4}} .
$$

Here, the function $\varphi_{\mu}$ is defined by $\varphi_{\mu}(\mu \operatorname{sech} \alpha)=\alpha-\tanh \alpha$.

We split the proof of Proposition A.1 into two parts. The first part (which is analogous to Lemma 6 in [2]) is

Lemma A.3. Let $q>0$ and $\rho>-1$ such that

$$
\frac{q}{2}>\rho+1, \quad \frac{q}{3} \geq \rho+\frac{1}{3} .
$$


Then

$$
\sup _{\mu \geq 1 / 2}\left(\int_{0}^{\infty}\left|J_{\mu}(r)\right|^{q} r^{\rho} d r+\int_{\mu-\mu^{1 / 3}}^{\infty}\left|H_{\mu}^{(1)}(r)\right|^{q} r^{\rho} d r\right)<\infty .
$$

Arguing slightly more carefully, we can replace the lower bound $\rho>-1$ by $\frac{q}{2}+\rho+1>$ 0 . More generally, it can be improved to $\mu_{0} q+\rho+1>0$ if we restrict the supremum to $\mu \geq \mu_{0} \geq 1 / 2$. This is only needed to ensure the integrability of $\left|J_{\mu}(r)\right|^{q} r^{\rho}$ near $r=0$.

Proof of Lemma A.3. We are going to use the upper bounds from Proposition A.2. Since they coincide for $J_{\mu}$ and $H_{\mu}^{(1)}$ in the range $r \geq \mu-\mu^{1 / 3}$, we only prove the lemma for $J_{\mu}$. We write $\int_{0}^{\infty}\left|J_{\mu}(r)\right|^{q} r^{\rho} d r=I_{1}+I_{2}+I_{3}+I_{4}+I_{5}+I_{6}$, where the different terms correspond to the following regions of integration:

$$
\begin{array}{ll}
I_{1}: & 0<r \leq 1 \\
I_{2}: & 1<r \leq \mu \operatorname{sech} \alpha_{0}, \\
I_{3}: & \mu \operatorname{sech} \alpha_{0}<r \leq \mu-\mu^{1 / 3}, \\
I_{4}: & \mu-\mu^{1 / 3}<r \leq \mu+\mu^{1 / 3} \\
I_{5}: & \mu+\mu^{1 / 3}<r \leq 2 \mu, \\
I_{6}: & r>2 \mu .
\end{array}
$$

In each of the regions we use the bounds from Proposition A.2 and we only make a few remarks about the straightforward computations. The finiteness of $I_{1}$ requires $q \mu+\rho+1>0$, which follows from $\rho>-1$. To bound $I_{2}$ we use the fact that $\left|J_{\mu}(r)\right| \leq C \mu^{-1}$ for $0<r \leq \mu \operatorname{sech} \alpha_{0}$, which is an easy consequence of Proposition A.2. To bound $I_{3}$ we split the region of integration into intervals $\left(\mu-2^{j+1} \mu^{1 / 3}, \mu-2^{j} \mu^{1 / 3}\right.$ ] and use $\varphi_{\mu}(r) \geq \varphi_{\mu}\left(\mu-2^{j} \mu^{1 / 3}\right) \geq C^{-1} \mu^{-1} 2^{3 j / 2}$ in each such interval. This yields $I_{3} \leq C \mu^{-q / 3+\rho+1 / 3}$, which is uniformly bounded in $\mu$ by assumption. We obtain the same bound on $I_{4}$ and, if $q>4$, on $I_{5}$. Finally, if $q / 2-\rho-1>0$ then $I_{6}$ is finite and satisfies $I_{6} \leq C \mu^{-q / 2+\rho+1}$. The same bound holds for $I_{5}$ if $q<4$ and, with a factor of $\ln \mu$, if $q=4$. This concludes the sketch of the proof.

The second part in the proof of Proposition A.1 (which is analogous to equation (2.28) in [2]) is

Lemma A.4. Let $q>0$ and $\rho>-1$ such that

$$
\frac{q}{2}>\rho+1, \quad \frac{q}{3} \geq \rho+\frac{1}{3} .
$$

Then

$$
\sup _{\mu \geq 1 / 2} \int_{0}^{\mu-\mu^{1 / 3}} \int_{r}^{\mu-\mu^{1 / 3}}\left|J_{\mu}(r)\right|^{q}\left|H_{\mu}^{(1)}\left(r^{\prime}\right)\right|^{q}\left(r r^{\prime}\right)^{\rho} d r^{\prime} d r<\infty
$$


Proof of Lemma A.4. We decompose the double integral as $I_{1}+I_{2}$, corresponding to the following regions of integration:

$$
\begin{aligned}
& I_{1}: \quad 0<r \leq \mu \operatorname{sech} \alpha_{0}, r<r^{\prime} \leq \mu-\mu^{1 / 3} \\
& I_{2}: \quad \mu \operatorname{sech} \alpha_{0}<r \leq \mu-\mu^{1 / 3}, r<r^{\prime} \leq \mu-\mu^{1 / 3}
\end{aligned}
$$

To bound $I_{1}$ we use the fact that $r\left|H_{\mu}^{(1)}(r)\right|^{2}$ is a decreasing function of $r$ [36, p. 446] and obtain for $q / 2>\rho+1$,

$$
\int_{r}^{\mu-\mu^{1 / 3}}\left|H_{\mu}^{(1)}\left(r^{\prime}\right)\right|^{q}\left(r^{\prime}\right)^{\rho} d r^{\prime} \leq r^{q / 2}\left|H_{\mu}^{(1)}(r)\right|^{q} \int_{r}^{\infty}\left(r^{\prime}\right)^{\rho-q / 2} d r^{\prime}=\frac{r^{\rho+1}}{q / 2-\rho-1}\left|H_{\mu}^{(1)}(r)\right|^{q} .
$$

The bounds from Proposition A.2 show that $\left|J_{\mu}(r)\right|\left|H_{\mu}^{(1)}(r)\right| \leq C^{2} \mu^{-1}$ for $0<r \leq$ $\mu \operatorname{sech} \alpha_{0}$, and therefore

$$
I_{1} \leq \frac{C^{2 q} \mu^{-q}}{q / 2-\rho-1} \int_{0}^{\mu-\mu^{1 / 3}} r^{2 \rho+1} d r \leq C^{\prime} \mu^{-q+2 \rho+2} .
$$

This is uniformly bounded since $q / 2>\rho+1$.

To bound $I_{2}$ we argue similarly, but we estimate slightly differently

$$
\int_{r}^{\mu-\mu^{1 / 3}}\left|H_{\mu}^{(1)}\left(r^{\prime}\right)\right|^{q}\left(r^{\prime}\right)^{\rho} d r^{\prime} \leq r^{q / 2}\left|H_{\mu}^{(1)}(r)\right|^{q} \int_{r}^{\mu}\left(r^{\prime}\right)^{\rho-q / 2} d r^{\prime} \leq r^{\rho}(\mu-r)\left|H_{\mu}^{(1)}(r)\right|^{q} .
$$

Proposition A.2 yields $\left|J_{\mu}(r)\right|\left|H_{\mu}^{(1)}(r)\right| \leq C^{2} \mu^{-1 / 2}(\mu-r)^{-1 / 2}$ for $\mu \operatorname{sech} \alpha_{0}<r \leq$ $\mu-\mu^{1 / 3}$, and therefore

$$
I_{2} \leq C^{2 q} \mu^{-q / 2} \int_{\mu \operatorname{sech} \alpha_{0}}^{\mu-\mu^{1 / 3}}(\mu-r)^{1-q / 2} r^{2 \rho} d r \leq C_{q} \mu^{2 \rho-q / 2} \int_{\mu \operatorname{sech} \alpha_{0}}^{\mu-\mu^{1 / 3}}(\mu-r)^{1-q / 2} d r .
$$

We conclude that

$$
I_{2} \leq C_{q}^{\prime} \times \begin{cases}\mu^{2 \rho-2 q / 3+2 / 3} & \text { if } q>4 \\ \mu^{2 \rho-2} \ln \mu & \text { if } q=4 \\ \mu^{2 \rho-q+2} & \text { if } q<4\end{cases}
$$

Under our assumptions on $q$ and $\rho$, this is uniformly bounded, as claimed.

Finally, we give the

Proof of Proposition A.1. Let $\rho=-q(\nu-2) / 2+\nu-1$. The conditions $q<2 \nu /(\nu-2)$ and $q>2 \nu /(\nu-1)$ imply $\rho>-1$ and $q / 2>\rho+1$, respectively. Finally, the condition $q / 3 \geq \rho+1 / 3$ follows from $q>2 \nu /(\nu-1)$ and $\nu \geq 2$. Therefore we can apply 
Lemmas $\mathrm{A.3}$ and $\mathrm{A.4}$ and find that

$$
\begin{aligned}
\int_{0}^{\infty} & \int_{r}^{\infty}\left|J_{\mu}(r)\right|^{q}\left|H_{\mu}^{(1)}(r)\right|^{q}\left(r r^{\prime}\right)^{-q(\nu-2) / 2+\nu-1} d r d r^{\prime} \\
= & \int_{0}^{\mu-\mu^{1 / 3}} \int_{r}^{\mu-\mu^{1 / 3}}\left|J_{\mu}(r)\right|^{q}\left|H_{\mu}^{(1)}(r)\right|^{q}\left(r r^{\prime}\right)^{-q(\nu-2) / 2+\nu-1} d r d r^{\prime} \\
& +\int_{0}^{\infty} \int_{\max \left\{r, \mu-\mu^{1 / 3}\right\}}^{\infty}\left|J_{\mu}(r)\right|^{q}\left|H_{\mu}^{(1)}(r)\right|^{q}\left(r r^{\prime}\right)^{-q(\nu-2) / 2+\nu-1} d r d r^{\prime}
\end{aligned}
$$

is uniformly bounded in $\mu \geq 1 / 2$. The fact that the integrals are uniformly bounded for $0 \leq \mu \leq 1 / 2$ follows immediately from standard results about Bessel functions. This concludes the proof of the proposition.

\section{REFERENCES}

[1] A. A. Abramov, A. Aslanyan, E. B. Davies, Bounds on complex eigenvalues and resonances. J. Phys. A 34 (2001), 57-72.

[2] J. A. Barcelo, A. Ruiz, L. Vega, Weighted estimates for the Helmholtz equation and some applications. J. Funct. Anal. 150 (1997), 356-382.

[3] A. Borichev, L. Golinskii, S. Kupin, A Blaschke-type condition and its application to complex Jacobi matrices. Bull. London Math. Soc. 41 (2009), 117-123.

[4] E. A. Carlen, R. L. Frank, E. H. Lieb, Stability estimates for the lowest eigenvalue of a Schrödinger operator. Geom. Funct. Anal. 24 (2014), no. 1, 63-84.

[5] E. B. Davies, J. Nath, Schrödinger operators with slowly decaying potentials. J. Comput. Appl. Math. 148 (2002), 1-28.

[6] M. Demuth, M. Hansmann, G. Katriel, Eigenvalues of non-selfadjoint operators: A comparison of two approaches, in: Mathematical Physics, Spectral Theory and Stochastic Analysis, Springer, 2013, 107-163.

[7] I. Egorova, L. Golinskiu,, On the location of the discrete spectrum for complex Jacobi matrices. Proc. Amer. Math. Soc. 133 (2005), no. 12, 3635-3641.

[8] R. L. Frank, Eigenvalue bounds for Schrödinger operators with complex potentials. Bull. Lond. Math. Soc. 43 (2011), no. 4, 745-750.

[9] R. L. Frank, A. Laptev, E. H. Lieb, R. Seiringer, Lieb-Thirring inequalities for Schrödinger operators with complex-valued potentials. Lett. Math. Phys. 77 (2006), 309-316.

[10] R. L. Frank, A. Pushnitski, Trace class conditions for functions of Schrödinger operators. Comm. Math. Phys. 335 (2015), no. 1, 477-496.

[11] R. L. Frank, J. Sabin, Restriction theorems for orthonormal functions, Strichartz inequalities and uniform Sobolev estimates. Preprint (2014): http://arxiv.org/pdf/1404.2817.pdf

[12] L. B. Golinskiı̌, I. E. Egorova, On limit sets for the discrete spectrum of complex Jacobi matrices. (Russian) Mat. Sb. 196 (2005), no. 6, 43-70; translation in Sb. Math. 196 (2005), no. 5-6, 817844.

[13] K. Guo, A uniform $L^{p}$ estimate of Bessel functions and distributions supported on $S^{n-1}$. Proc. Amer. Math. Soc. 125 (1997), no. 5, 1329-1340.

[14] A. D. Ionescu, D. Jerison, On the absence of positive eigenvalues of Schrödinger operators with rough potentials. Geom. Funct. Anal. 13 (2003), 1029-1081.

[15] J. B. Keller, Lower bounds and isoperimetric inequalities for eigenvalues of the Schrödinger equation. J. Math. Phys. 2 (1961), 262-266. 
[16] C. E. Kenig, A. Ruiz, C. D. Sogge, Uniform Sobolev inequalities and unique continuation for second order constant coefficient differential operators. Duke Math. J. 55 (1987), 329-347.

[17] H. Koch, D. Tataru, Carleman estimates and absence of embedded eigenvalues. Comm. Math. Phys. 267 (2006), no. 2, 419-449.

[18] A. Laptev, O. Safronov, Eigenvalue estimates for Schrödinger operators with complex potentials. Comm. Math. Phys. 292 (2009), 29-54.

[19] E. H. Lieb, W. Thirring, Inequalities for the moments of the eigenvalues of the Schrödinger Hamiltonian and their relation to Sobolev inequalities. Studies in Mathematical Physics. Princeton University Press, Princeton (1976), pp. 269-303.

[20] R. M. Martirosjan, On the spectrum of the non-selfadjoint operator $-\Delta u+c u$ in three dimensional space. (Russian) Izv. Akad. Nauk Armyan. SSR. Ser. Fiz.-Mat. Nauk 10 (1957), no. 1, 85-111.

[21] R. M. Martirosjan, On the spectrum of various perturbations of the Laplace operator in spaces of three or more dimensions. (Russian) Izv. Akad. Nauk SSSR Ser. Mat. 24 (1960), 897-920.

[22] S. N. Naboko, On the dense point spectrum of Schrödinger and Dirac operators. Teoret. Mat. Fiz. 68 (1986), no. 1, 18-28.

[23] M. A. Naumark, Investigation of the spectrum and the expansion in eigenfunctions of a nonselfadjoint operator of the second order on a semi-axis. (Russian) Trudy Moskov. Mat. Obšč. 3 (1954), 181-270.

[24] B. S. Pavlov, On a non-selfadjoint Schrödinger operator. (Russian) 1966 Probl. Math. Phys., No. I, Spectral Theory and Wave Processes (Russian) pp. 102-132 Izdat. Leningrad. Univ., Leningrad

[25] B. S. Pavlov, On a non-selfadjoint Schrödinger operator. II. (Russian) 1967 Problems of Mathematical Physics, No. 2, Spectral Theory, Diffraction Problems (Russian) pp. 133-157 Izdat. Leningrad. Univ., Leningrad

[26] B. S. Pavlov, On a non-selfadjoint Schrödinger operator. III. (Russian) Problems of mathematical physics, No. 3: Spectral theory (Russian), pp. 59-80. Izdat. Leningrad. Univ., Leningrad, 1968.

[27] M. Reed, B. Simon, Methods of modern mathematical physics. IV. Analysis of operators. Academic Press [Harcourt Brace Jovanovich Publishers], New York, 1978.

[28] O. Safronov, Estimates for eigenvalues of the Schrödinger operator with a complex potential. Bull. Lond. Math. Soc. 42 (2010), no. 3, 452-456.

[29] B. Simon, Some Schrödinger operators with dense point spectrum. Proc. Amer. Math. Soc. 125 (1997), no. 1, 203-208.

[30] S. A. Stepin, On spectral components of the Schrödinger operator with a complex potential. (Russian) Uspekhi Mat. Nauk 68 (2013), no. 1 (409), 199-200; translation in Russian Math. Surveys 68 (2013), no. 1, 186-188.

[31] S. A. Stepin, Estimate for the number of eigenvalues of the nonselfadjoint Schrödinger operator. (Russian) Dokl. Akad. Nauk 455 (2014), no. 4, 394-397; translation in Dokl. Math. 89 (2014), no. 2, 202-205.

[32] R. Strichartz, Restrictions of Fourier transforms to quadratic surfaces and decay of solutions of wave equations. Duke Math. J. 44 (1977), 705-714.

[33] E. C. Titchmarsh, Eigenfunction expansions associated with second-order differential equations, Part I. Second Edition, Clarendon Press, Oxford 1962.

[34] L. Vega, Restriction theorems and the Schrödinger multiplier on the torus. In: Partial differential equations with minimal smoothness and applications (Chicago, IL, 1990), 199-211, IMA Vol. Math. Appl. 42, Springer, New York, 1992.

[35] J. von Neumann, E. P. Wigner, Über merkwürdige diskrete Eigenwerte. Z. Phys. 30 (1929), $465-467$. 
[36] G. N. Watson, A treatise on the theory of Bessel functions. Cambridge Univ. Press, Cambridge, UK, 1944.

Rupert L. Frank, Mathematics 253-37, Caltech, Pasadena, CA 91125, USA

E-mail address: rlfrank@caltech.edu

Barry Simon, Mathematics 253-37, Caltech, Pasadena, CA 91125, USA

E-mail address: bsimon@caltech.edu 